\title{
Hernia uterine inguinale with endometriosis in adult Mayer Rokintasky Kuster Hauser syndrome
}

\author{
Padmasri $R^{1}$, Rupa S Iyengar ${ }^{1}$
}

\begin{abstract}
A young 25 year old woman presented with primary infertility and was found to have a big left inguinal mass with a blind vagina. Based on the findings of magnetic resonance imaging, a history of probable primary amenorrhea and absence of cervix on clinical examination she was diagnosed as MRKH with inguinal herniation of uterus and adnexae. Operative findings showed bilateral grossly distorted inguinal masses with evidence of endometriosis. Postoperative pathology findings demonstrated endometriosis.
\end{abstract}

\section{Introduction}

The Mayer-Rokitansky-Küster-Hauser (MRKH) syndrome is characterized by congenital aplasia of the uterus and the upper part $(2 / 3)$ of the vagina in women showing normal development of secondary sexual characteristics and a normal 46, XX karyotype. It affects at least 1 out of 4500 women $^{1}$. The majority of cases appear to be sporadic, however family cases have also been described. The mode of inheritance seems to be autosomal dominant with an incomplete degree of penetrance and variable expressivity. Type I (isolated) MRKH is less frequent than Type II $^{2}$. Several cases have been reported where the tubes and ovaries have herniated into the inguinal canal. Most cases concern the paediatric population with a congenital hernia sac. Entrapment of adnexae in an indirect inguinal hernia is rare in adult women ${ }^{3}$.

\section{Case report}

A 25 year old lady, born to nonconsanguinous parents, came to us

${ }^{1}$ Sapthagiri Institute of Medical Sciences and Research Centre, Bangalore, India.

Correspondence: R Padmasri

E-mail: drpadmasuraj@gmail.com with history of primary infertility and secondary amenorrhoea, apparently having attained menarche at 16 years, had regular periods until six months back. There was no history of dysmenorrhoea. She was married and sexually active since three years. Her secondary sexual characters were well developed. External genitalia were female. Local examination showed a palpable mass in left inguinal region $-8 \times 7 \mathrm{~cm}$. The mass was non tender, irreducible, and cystic in consistency (Figure 1). Per speculum showed a blind vaginal pouch $4 \mathrm{cms}$ depth. Cervix was not seen. Per vaginum examination confirmed speculum examination. No uterus or appendages felt in midline. Thyroid profile, prolactin, follicle stimulating hormone, luteinising hormone, testosterone, dihydroepiandrosterone sulphate levels were within normal limits. Cytogenetic report was normal female karyotype of 46 XX. MRI imaging showed a case of Mayer-Rokintansky-Kuster-Hauser syndrome showing wide separation of hypoplastic uterine horns containing endometrium with herniation of these uterine horns in to bilateral inguinal regions with respective ovary and dilated blood filled fallopian tubes. Left sided mass of $15 \times 5.8 \mathrm{~cm}$. Right sided mass measuring $8.8 \times 5.7 \mathrm{~cm}$. (Figure 2). Transrectal USG study showed blind vagina measuring about $4.5 \mathrm{~cm}$ in length. Renal and skeletal systems were normal. On surgery a right sided endometriotic tubo ovarian mass was found stuck to the right lateral pelvic wall, in the region of the right internal iliac ring. The rudimentary horn was noticed

\section{Figure 1. Left inguinal swelling.}

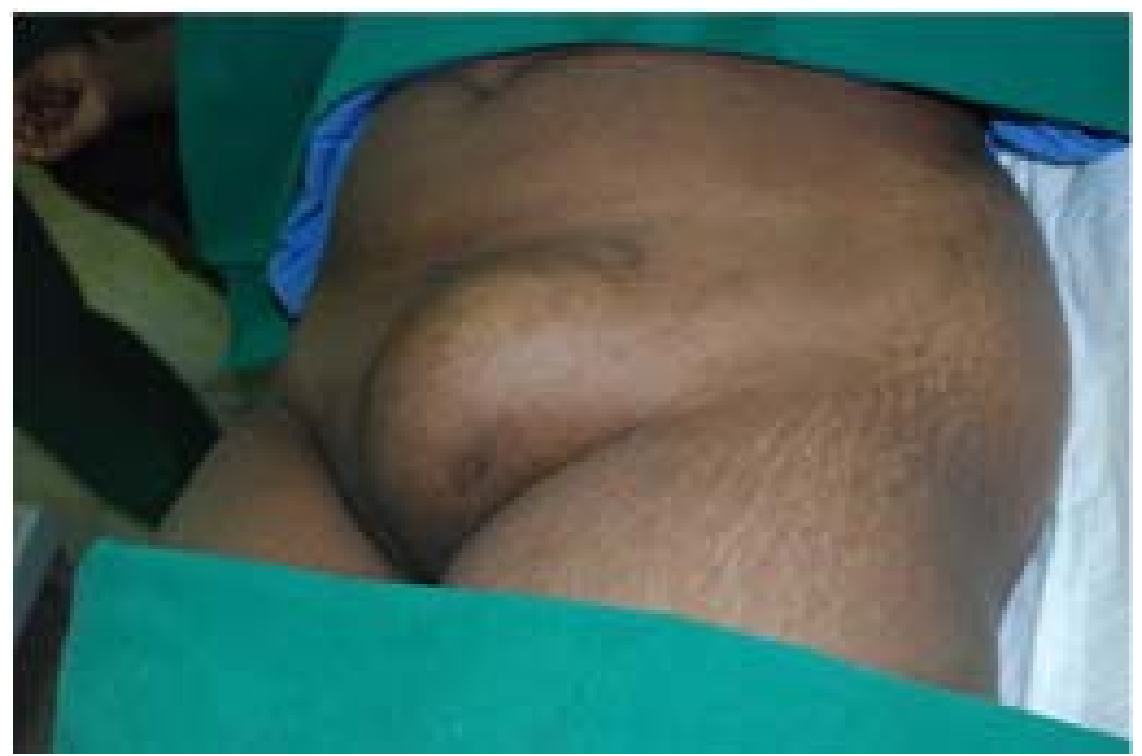




\section{Figure 2. MRI - Rudimentary uterine horn with endometrium with dilated blood filled fallopian tubes.}

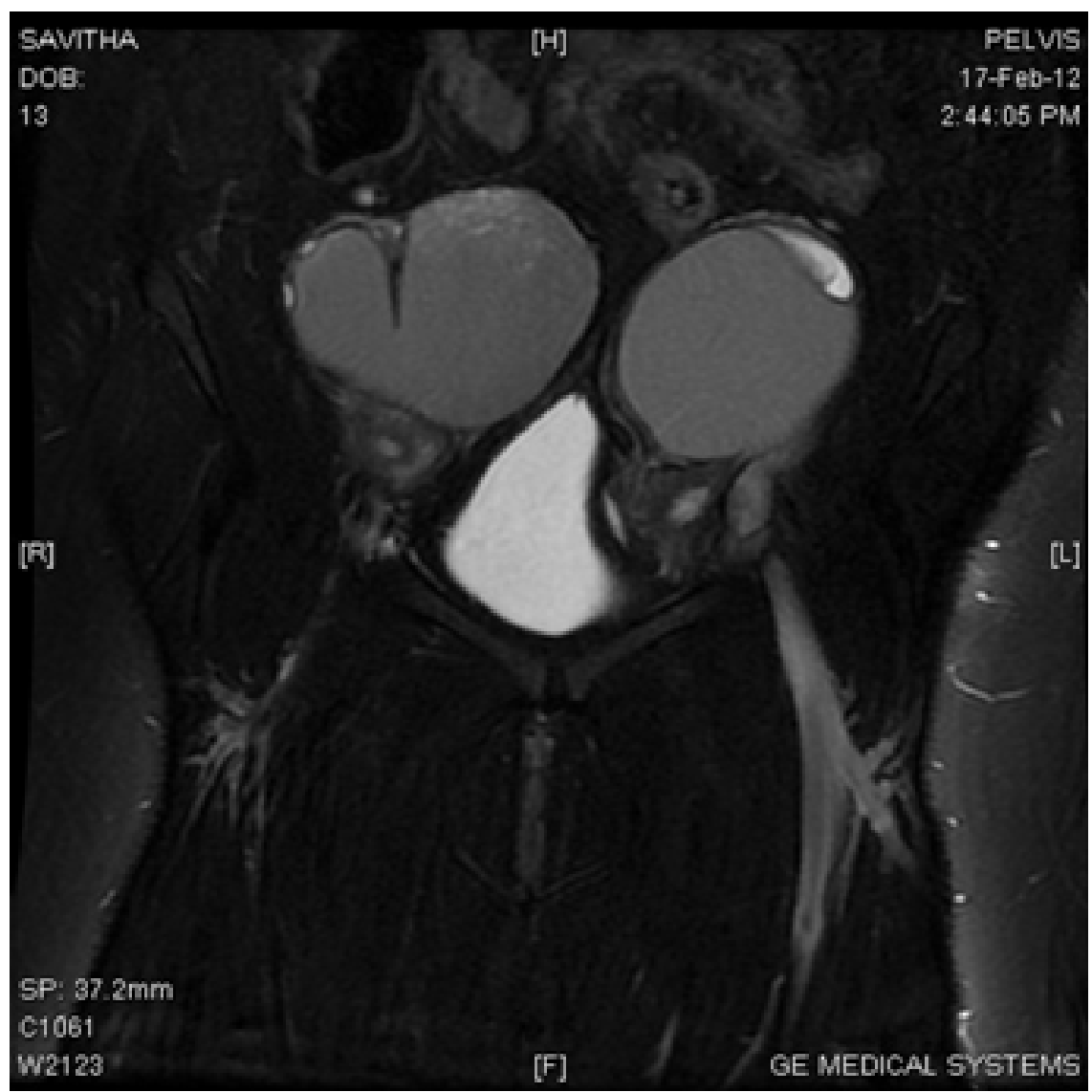

\section{Figure 3. Inguinal incision for removal of left uterine horn and tubo ovarian mass.}

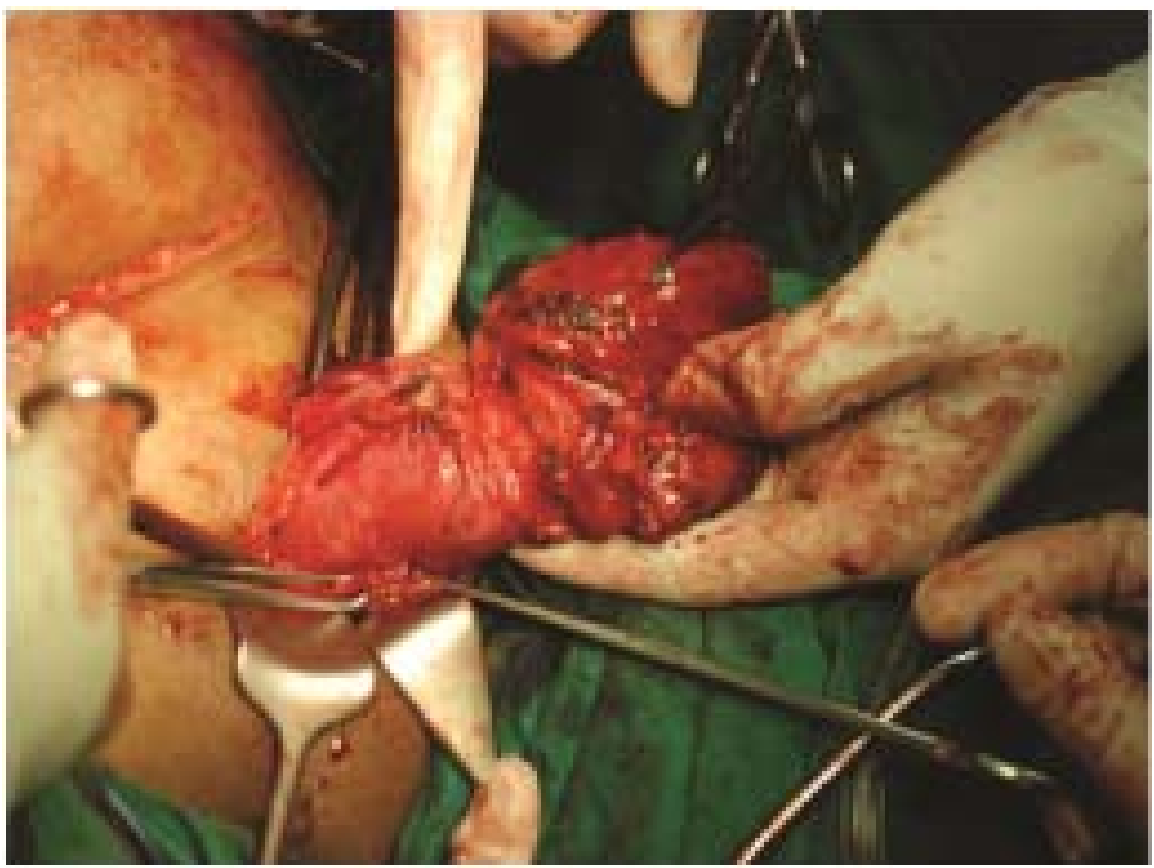

beneath the endometriotic cyst. The tube was distended to form a huge haematosaphinx which had partially prolapsed into the right inguinal canal. The contents were reduced by traction and blunt dissection. The right rudimentary horn was excised with the tubo ovarian mass. On the left side a bigger mass was seen with major portion of the mass extending into left inguinal canal which could not be reduced. Hence a left inguinal incision was taken and a left inguinal hysterectomy was done (Figure 3). Conservative surgery could not be done due to gross distortion of structures. Histopathology of the mass showed rudimentary horn of uterus with adenomyosis, chocolate cyst of ovary compatible with endometriosis and dilated fallopian tube showing hemosiderin laden macrophage, chronic inflammatory cells, congested blood vessels and fibroblast, giant cells. No cervical tissue was seen.

\section{Discussion}

Inguinal hernia containing uterus and endometriosis is exceedingly rare. Most inguinal endometriosis is located at an extra pelvic site near the round ligament. One such reported case of a patient with inguinal hernia containing rudimentary uterine horn and endometriosis was unilateral ${ }^{4}$. A handful of other cases show a unilateral herniation of uterus, tubes and ovaries without endometriosis ${ }^{5,6}$. Several cases of ovarian endometriosis in MRKH without uterine hernia have been reported ${ }^{7,8}$. Our patient was found to have bilateral herniation and endometriosis. Endometriosis in a patient with MRKH syndrome with a small rudimentary uterine horns and functional endometrium can be assumed to have developed by retrograde menstruation theory. Such patients usually present with cyclical pain during menstruation as reported in the one case $^{4}$. Surprisingly our patient did not have any pain nor did she acknowledge the evident primary amenorrhoea. Psychosocial elements should be considered when dealing with 
such patients. Hernia uterine inguinal has been reported most commonly in the literature as both persistent müllerian duct syndrome and male pseudohermaphroditism. It is most often seen in a phenotypically normal male infant having both testes and uterine tissue present. Few cases have been documented to occur in the female sex or the adult patient. This subset may be at higher risk for hernia uterine inguinale, and if presenting with complaints of pain or inguinal mass, it should likewise be considered in the differential diagnosis. Abdominal and pelvic imaging is useful in the diagnosis of this condition because it may aid in identifying patients with coexisting mullerian malformations ${ }^{9}$.

\section{REFERENCES}

1. Morcel K, Camborieux L. MayerRokitansky-Küster-Hauser (MRKH) syndrome. Orphanet J Rare Dis. 2007; 2: 13.

2. Guerrier D, Mouchel T, Pasquier L, Pellerin I. The Mayer-Rokitansky-Küster-Hauser syndrome (congenital absence of uterus and vagina) - phenotypic manifestations and genetic approaches. J Negat Results Biomed. 2006; 5: 1.

3. Machado NO, Machado NN. Unusual contents of inguinal hernia sac. An approach to management. Surgical Science. 2011; 2: 322-5.

4. Kamio $M$, Nagata $T$, Yamasaki $H$, Yoshinaga M, Douchi T. Inguinal hernia containing functioning, rudimentary uterine horn and endometriosis. Obstet Gynecol. 2009; 113: 563-6.

5. Kriplani A, Banerjee N, Aminni AC, Kucheria
$\mathrm{K}$, Takkar D. Hernia uterus inguinale in a 46, XX female. A case report. J Reprod Med. 2000; 45(1): 48-50.

6. Mandel DC, Beste T, Hope W. Hernia uterine inguinale: an uncommon cause of pelvic pain in the adult female patient. $\mathrm{J}$ Minim Invasive Gynecol. 2010; 17(6): 787-90.

7. Goluda M, St Gabrys M, Ujec M, Jedryka M, Goluda C. Bicornuate rudimentary uterine horns with functioning endometrium and complete cervical-vaginal agenesis coexisting with ovarian endometriosis: a case report. Fertil Steril. 2006; 86(2): 462. 9-11. 8.

8. Cho MK, Kim CH, Oh ST. Endometriosis in a patient with Rokitansky-Kuster-Hauser syndrome. J Obstet Gynaecol Res. 2009; 35(5): 994-6.

9. Odi TO, Abdur-Rahman LO, Nasir AA. Persistent Mullerian duct syndrome: a case report and review of the literature. Afr $\mathrm{J}$ Paediatr Surg. 2010; 7(3): 191-3. 